\title{
Quantum Networks: Dynamics of Open Nanostructures
}

\author{
GÜNTER MAHLER and RAINER WAWER* \\ Institut für Theoretische Physik, Universität Stuttgart, Pfaffenwaldring 57, 70550 Stuttgart, Germany
}

The superposition principle makes quantum networks behave very differently from their classical counterparts: We discuss how local and non-local coherence are generated and how these may affect the function of composite systems. Numerical examples concern quantum trajectories, quantum noise and quantum parallelism.

Keywords: Coherence, entanglement, quantum trajectories, quantum parallelism

\section{GENERAL BACKGROUND}

Beyond some 15 years from now any realistic roadmap for nanoelectronics [1] tends to become rather vague: The present trend towards miniaturization is expected to continue till then, but as physics is not scale-invariant, fundamental limitations will be piling up, all of which are eventually related to quantum features of some sort: there are limits of manufacturing, limits of control, limits of simulation capabilities, limits of classical system theory, and limits of classical models of computation, to name but a few.

We will not be able to discuss these issues here at any depth; instead, we intend to address some pertinent questions based on "toy-models" rather than sophisticated device simulations. Nevertheless, this approach should be able to demonstrate in what sense quantum networks will differ from classical ones.

\subsection{How Can We Design Quantum Systems?}

Stable structure, though eventually a quantum phenomenon in its own right, enters nanophysics as a "classical" design tool: Confinement of electron-(or photon-) fields allows to discretize the state space, at least in some energy range of interest. This "quantum-size-effect" considerably simplifies the specification and observation of the non-classical features to be discussed below, as it significantly reduces the number of "subsystems" to be considered.

\subsection{What is a Subsystem?}

It turns out that the only viable definition of a subsystem is operational: A subsystem has to be separated out by the classical environment (experimental set-up) in terms of measurement modes. A quantum dot or an atom (within an array) will be a

\footnotetext{
* Corresponding author.
} 
subsystem if and only if it is selectively "contacted". Note that if this condition is not fulfilled, the array has to be treated as a unit; though this will not change the total state space, it will certainly change the type of possible observations.

\subsection{How do we Describe Subsystems?}

We restrict ourselves to subsystems $\mu$ characterized by $n$ different states $\left|p_{\mu}\right\rangle$, each. The number of independent observables (represented by a set of orthonormalized operators $\left.\hat{\lambda}_{j}(\mu)\right)$ is then restricted to $n^{2}$, i.e., $j=0$ (denoting the unit-operator: "no action"), $1, \ldots, n^{2}-1$. Any operator can be written as some linear combination of these. We may choose the $\hat{\lambda}_{j}$ such that the expectation-values $\left\langle\hat{\lambda}_{j}\right\rangle$ form a vector [2], [3], for $n=2$ the so-called Blochvector.

\subsection{How do we Describe Networks?}

Any operator (for a network composed of $N$ subsystems, say) can be written as a linear combination of the $n^{2 N}$ "cluster-operators"

$$
\hat{Q}_{j k \ldots r}=\hat{\lambda}_{j}(1) \hat{\lambda}_{k}(2) \ldots \hat{\lambda}_{r}(N)
$$

These $\hat{Q}$, in general, do not commute; they come in different classes, specified by the number $m \leq N$ of subsystems which are actually affected (which equals the number of indices unequal zero): $\hat{Q}_{00 \ldots 0}$ is the unit-operator, $\hat{Q}_{j 0 k \ldots 0}$ an operator acting on $\mu=1,3$ (compact notation $\hat{\lambda}_{j}(1) \hat{\lambda}_{k}(3)$ ), etc. It is important to realize that our definition of subsystems guarantees that expectation values $\langle\hat{Q}\rangle$ of any such $m$-cluster-operator can be obtained from a series of respective coincidence experiments (ensemble- or time-averaged).

\subsection{Can we Reconstruct any State?}

The density matrix is uniquely specified once the expectation values of this full hierarchy of clusteroperators are given [3]. As the number of independent state parameters grows exponentially with $N$, complete state reconstruction [4] tends to be severely limited. So, $N$ needs to be small, as incomplete measurements are likely to miss nonclassical features all together. (This is why our world appears so classical).

\subsection{What is a "Classical" State?}

Within an entirely quantum mechanical treatment the notion of a "classical" state is somewhat ambiguous. For a definition one usually refers to a complete local basis as the eigenstates of a local operator (or set of those): For the two-level system studied here we use the local basis operators, $\hat{\lambda}_{3}(\mu)$. Products of their eigenstates, $\left|p_{1} p_{2}, \ldots, p_{N}\right\rangle$, $p_{i}=1,2, \ldots, n$, then define a complete basis set for the whole network, taken as our "classical" reference.

\subsection{What are "Non-classical" States?}

Superpositions of those classical states are now non-classical by definition: There are superpositions within a single subsystem only (local coherence, described here by the expectation values of $\left.\hat{\lambda}_{1}(\mu), \hat{\lambda}_{2}(\mu)\right)$ and superpositions involving more than one subsystem (entanglement, described by appropriate expectation values of $m \geq 2$ - cluster - operators). The fact that the subsystems are separable does not imply that the state of the network is separable into these constituents: The implies a kind of "quantum fuzziness" of the subsystems: Entangled states are states which, allowing for any local [5] unitary transformation, cannot be written as a simple product state. A useful entanglement measure should thus be invariant under such local transformations. (There is a generalization for mixed states).

\subsection{How can we Quantify Entanglement?}

Entanglement has been introduced as a property of a wavefunction not to factor into a product. This can be tested by means of appropriate 
expectation values. The matrix-elements [3]

$$
\boldsymbol{M}_{i j \ldots}^{\mu \nu \ldots}=\left\langle\left(\hat{\lambda}_{i}(\mu)-\left\langle\hat{\lambda}_{i}(\mu)\right\rangle\right)\left(\hat{\lambda}_{j}(\nu)-\left\langle\hat{\lambda}_{j}(\nu)\right\rangle\right) \cdots\right\rangle
$$

are of the form of covariances known from classical statistical physics; non-zero terms indicate deviations from the product state form [6]. Convenient measures, which are much less detailed but turn out to be invariant under any local unitary transformations, are (for $n=2$ )

$$
\beta^{\mu \nu}=\frac{1}{3} \sum_{i j} M_{i j}^{\mu \nu} M_{i j}^{\mu \nu}, \quad \beta^{\mu \nu \sigma}=\frac{1}{4} \sum_{i j k} M_{i j k}^{\mu \nu \sigma} M_{i j k}^{\mu \nu \sigma}
$$

These measures have upper bounds; here they are normalized to 1 . For a network with $N>2$ subsystems there is not just one but a whole hierarchy of such entanglement measures. In addition to these we also introduce a measure for local coherence; with the eigenstates of $\hat{\lambda}_{3}$ as the local reference $(n=2)$, a possible choice is

$$
\alpha^{\mu}=\left\langle\hat{\lambda}_{1}(\mu)\right\rangle^{2}+\left\langle\hat{\lambda}_{2}(\mu)\right\rangle^{2}
$$

Of course, $\alpha^{\mu}$ cannot be invariant under local unitary transformations.

\subsection{What is the Origin of Entanglement?}

Perhaps the best known source of entanglement is the permutation symmetry requirement for indistinguishable particles. However, such Fermi (Bose-) states are not characterized by specific entanglement types. Eigenstates of global operators like total angular momentum, may also exhibit entanglement. A dynamical source of entanglement, finally, can be traced back to any two-subsystem interaction (like Coulomb interaction): The corresponding Hamiltonian generates a unitary time evolution which is non-local and will thus, in general, change the entanglement.

\subsection{What are Observable Effects of Entanglement?}

While coherence physics can do with appropriate local measurements $(m=1)$, entanglement shows up not before $m \geq 2$-cluster-operators are involved: Typically this applies to the total Hamiltonian itself; well-known effects are energy shifts and (exchange-) splittings for Fermi-systems.

More specifically, entanglement-physics relates to the study of a single network through a number of selective modes or contacts. We will discuss three qualitatively different examples based on the same type of model.

\section{NUMERICAL SIMULATIONS}

\subsection{Outline of Model}

The model we are considering could be an array of $N=3$ quantum dots, here simplified as two-level subsystems. In order these to represent real subsystems, they need to be addressable separately. We assume they are spectroscopically different, i.e., they can be distinguished in frequency space and thus be separately driven by a classical external electromagnetic field (coupling strength $g^{\mu}$, detuning $\delta^{\mu}$ ). Their mutual coupling, $C_{R}^{\mu \nu}$, is of the Ising-spin type. This coupling modifies the energy level spacing of one system depending on the state of its neighbors. The eigenstates of this interacting network are still product states, though. Local coherence is introduced by the external driving fields, which is then transformed into entanglement by means of the interaction. In this way there is an entanglement source that can be switched on and off [3].

\subsection{Quantum Trajectories}

In the first two examples all three subsystems "play the same role", i.e., all the parameters $g, \delta$, $C_{F}$ are independent of $\mu$; they are chosen such that only the transitions from the ground state $|111\rangle$ to $|112\rangle,|121\rangle$ and $|211\rangle$ are in resonance. In addition 
there are 3 local damping channels, again all with the same damping rate $W$. The dynamics is described by the appropriate master equation.

As we are interested in the detailed simulation of individual networks, the theoretical approach of choice is stochastic unraveling (see [2] and references therein): In this method the master equation is interpreted as a rule for a modified non-unitary continuous evolution of a pure state, interrupted by stochastic jumps to another pure state. Incoherent driving is described as a selective rate-process (transition rates $\left(g^{\mu}\right)^{2} \pi / 2$ ) implying $\alpha^{\mu}=\beta^{\mu \nu}=\beta^{123}=0$ at all times ("classical case"). While the latter (Fig. 1a) is confined to the definite alternatives of being in the lower or upper state ("telegraph signal"), the trajectories in the nonclassical domain (Fig. 1b) are strongly correlated (they practically coincide here) and show a quite different behavior: Between the jumps there is a continuous motion, as coherence and entanglement allow to explore additional regions of state space: Figure 2 shows the time-averaged entanglement measures as they depend on $g / W$.
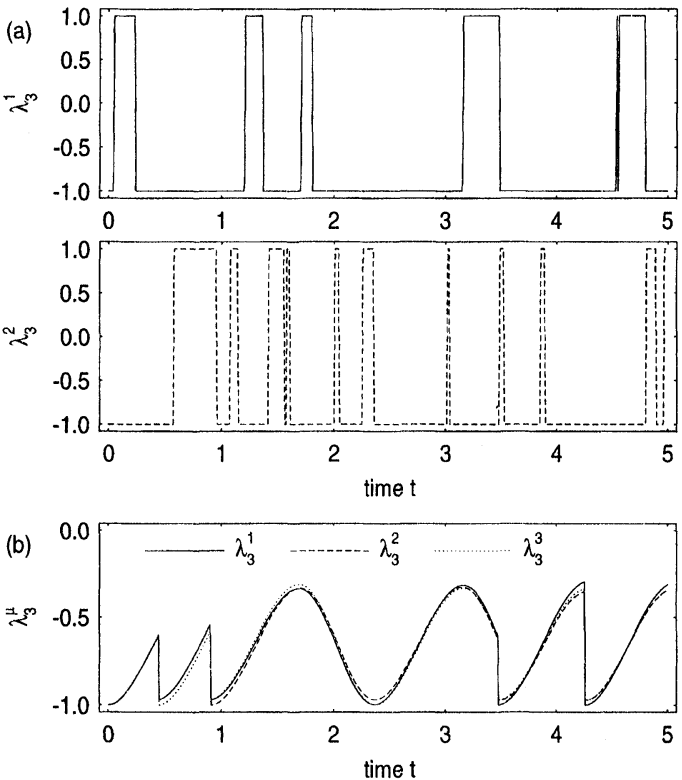

FIGURE 1 Quantum trajectories of a driven and damped $N=3$-network $(g / W=2.5)$ : (a) classical limit (incoherent pumping rate $=g^{2} \pi / 2$ ), (b) non-classical case (coherent driving).

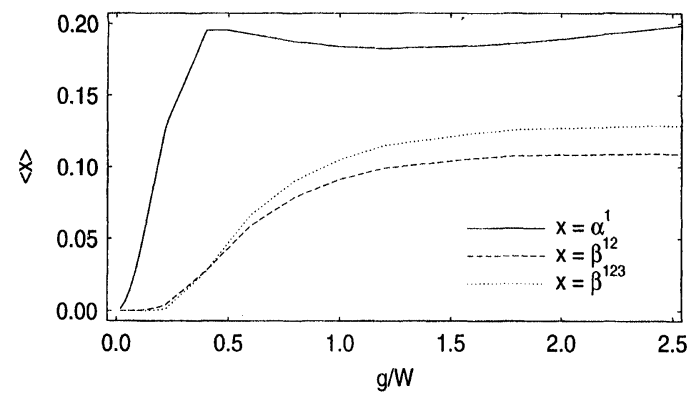

FIGURE 2 Averaged coherence-, $\alpha$, and entanglementmeasures, $\beta^{\mu \nu}, \beta^{123}$ as a function of the coherent driving field strength, $g$, over the damping rate, $W$.

\subsection{Quantum Noise}

The quantum trajectories as such do not constitute a measurement, let alone a complete one. However, assuming the damping of each subsystem to be due to radiative decay only, the trajectories of the above model directly connect to specific luminescence signals: Each photon detection event in one of the distinguishable frequency channels (photon counts $n^{\mu}$ per sampling time $T$ ) is related to a jump in the trajectory. The simulated detection traces may then be statistically analyzed in terms of the counting covariances [7].

$$
N^{\mu \nu \ldots}=\overline{\left(n^{\mu}-\overline{n^{\mu}}\right)\left(n^{\nu}-\overline{n^{\nu}}\right) \ldots}
$$

As shown in Figure 3, the noise properties, indeed, reflect the fact that the network state is (on average) non-classical and follows non-classical trajectories. The result for incoherent driving is

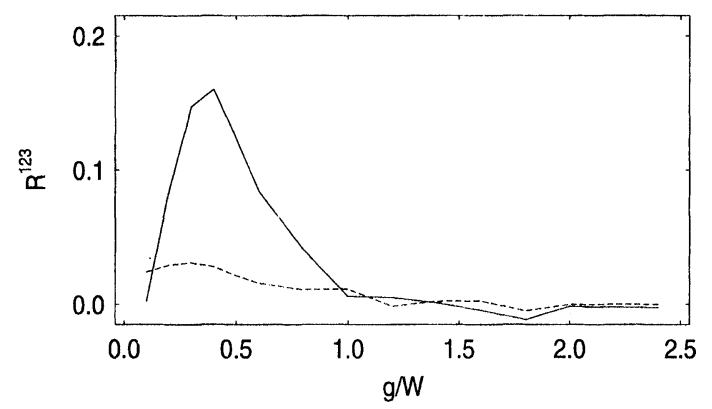

FIGURE 3 Photon-counting covariance of the luminescence signals: Shown is $R^{123}=N^{123} /\left(N^{11} N^{22} N^{33}\right)^{1 / 2}$ with $N^{\mu \nu \cdots}$ as defined in Eq. (5). The sampling time is $T \gg W^{-1}$. 
included for comparison (broken line); there is virtually no correlation $N^{123}$ in this case.

\subsection{Quantum Parallelsim}

Contrary to the two preceding examples we turn now to a closed system, with the sub-systems playing different roles: These roles are implemented by means of pulsed changes of the parameters controlling the Hamilton model: Well-known examples are $\pi$-pulses to invert the occupation of 2-level systems. Interacting networks of the type studied here allow to implement conditioned $\pi$ pulses, i.e., the action of the light pulse on subsystem $\mu=1$ is a $\pi$-pulse only, if the neighbor $\mu=2$ is in state $|1\rangle$, say; no action otherwise [8]. A continuously driven system 2 in contact at time $t_{1}$ with such a "memory-gate" 1 (pulse duration is assumed negligible), subsequently at $t_{2}$ in contact with a gate 3 will generate entanglement as discussed before. However, the "logic" of these gate operations make system 2 emulate an ensemble that was actually measured at those

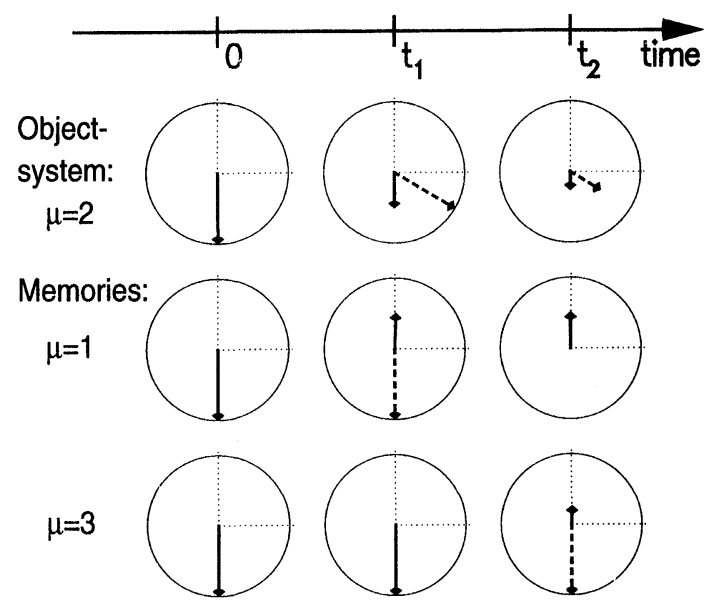

FIGURE 4 Single closed network: Subsystem-Bloch-vectors (in $\left\langle\hat{\lambda}_{2}\right\rangle,\left\langle\hat{\lambda}_{3}\right\rangle$-plane) at 3 instatnts of time. Subsystem $\mu=2$ is continuously driven (rotates counter-clockwise) and is brought into contact with a "gate"-subsystem $\mu=1$ at time $t_{1}$, and with a "gate" $\mu=3$ at time $t_{2}$ (broken lines: before gate operation). Each gate is seen to "project" subsystem 2 on the $\left\langle\hat{\lambda}_{3}\right\rangle$-axis, as in an ensemble measurement. Note that a single system measurement would result in either up or down. two times (Fig. 4): System 2 represents in parallel all potential trajectories going through the two measurement steps! This quantum parallelism exploits entanglement in a surprising way. Socalled quantum-algorithms would make sure that final measurements, which invariably pick an actual trajectory and thus deviate from ensemble behavior, indicate desired problem solutions [9]. Their reliance on large- $N$-quantum networks makes them a challenging target indeed for future developments.

\section{Acknowledgement}

Financial support by the Deutsche Forschungsgemeinschaft and by the Oregon Center of Optics is gratefully acknowledged. One of us (G.M.) thanks Howard Carmichael and Michael Raymer for valuable discussions.

\section{References}

[1] See, e.g., document from U.S. semiconductor industry, http://www.sematech.org/public/roadmap/doc.

[2] Keller, M. and Mahler, G. (1994). "Nanostructures, entanglement, and the physics of quantum control", $J$. Mod. Optics, 41, 2537; Mahler, G. and Weberruß, V. A. (1995). "Quantum Networks: Dynamics of Open Nanostructures", Springer, Berlin.

[3] Mahler, G., Keller, M. and Wawer, R. (1997). "Quantum networks: master equation and local measurements", $Z$. Phys. B, 104, 153.

[4] Leonhardt, U., Munroe, M., Kiss, T., Richter, Th. and Raymer, M. G. (1996). "Sampling of photon statistics and density matrix using homodyne detection", Optics Commun., 127, 144.

[5] A local unitary transformation can be written as a product of transformations on individual subsystems.

[6] cf. also the so-called Bell-inequalities: Bell, J. S. (1964). Physics (N.Y), 1, 195.

[7] Mahler, G. and Wawer, R. (1997). "Quantum dot arrays: preparation and detection of non-classical states", Superlattics and Microstructures, 21, 7.

[8] See e.g., Barenco, A., Deutsch, D. and Ekert, A. (1995). "Conditional quantum dynamics and logic gates", Phys. Rev. Letters, 74, 4083.

[9] Ekert, A. and Jozsa, R. (1996). Rev. mod. Physics, 68, 733.

\section{Authors' Biography}

Prof. Dr. Günter Mahler studied physics at the Universities of Frankfurt, Munich, and Regens- 
burg (Germany). He received his Ph.D. in 1972 and was appointed Professor for Theoretical Physics by the University of Stuttgart in 1978. He has been visiting Professor with the University of Strasbourg, France, the Arizona State University, the Santa $\mathrm{Fe}$ Institute, and the University of Oregon, USA. His main fields of interest concern semiconductor transport, molecular elec- tronics, quantum stochastics, quantum information, and quantum optics.

Rainer Wawer studied physics at the University of Stuttgart, where he received his Diploma in Theoretical Physics. He is presently working in the field of quantum measurement models and quantum trajectories in partial fulfillment of Ph.D. requirements. 

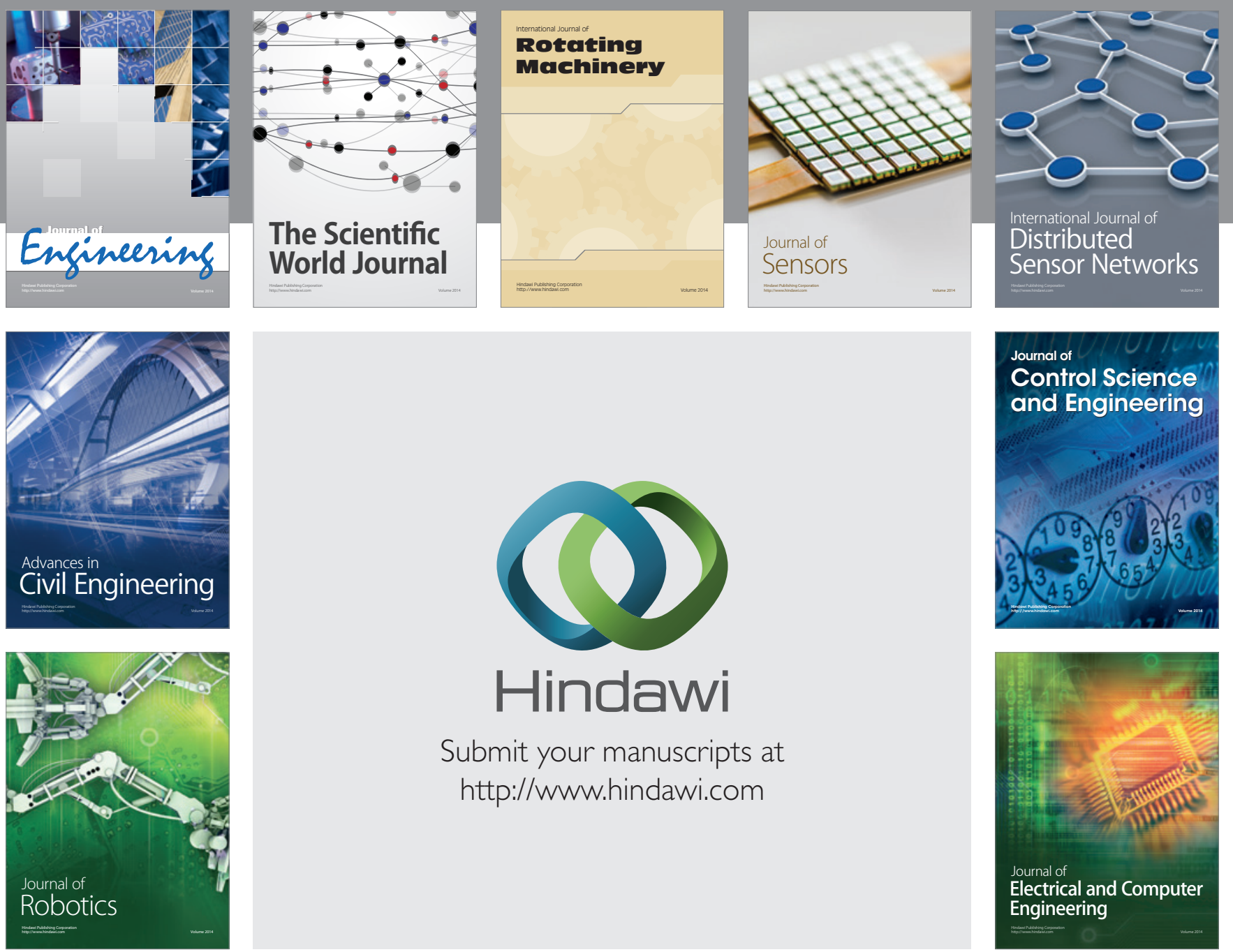

Submit your manuscripts at

http://www.hindawi.com
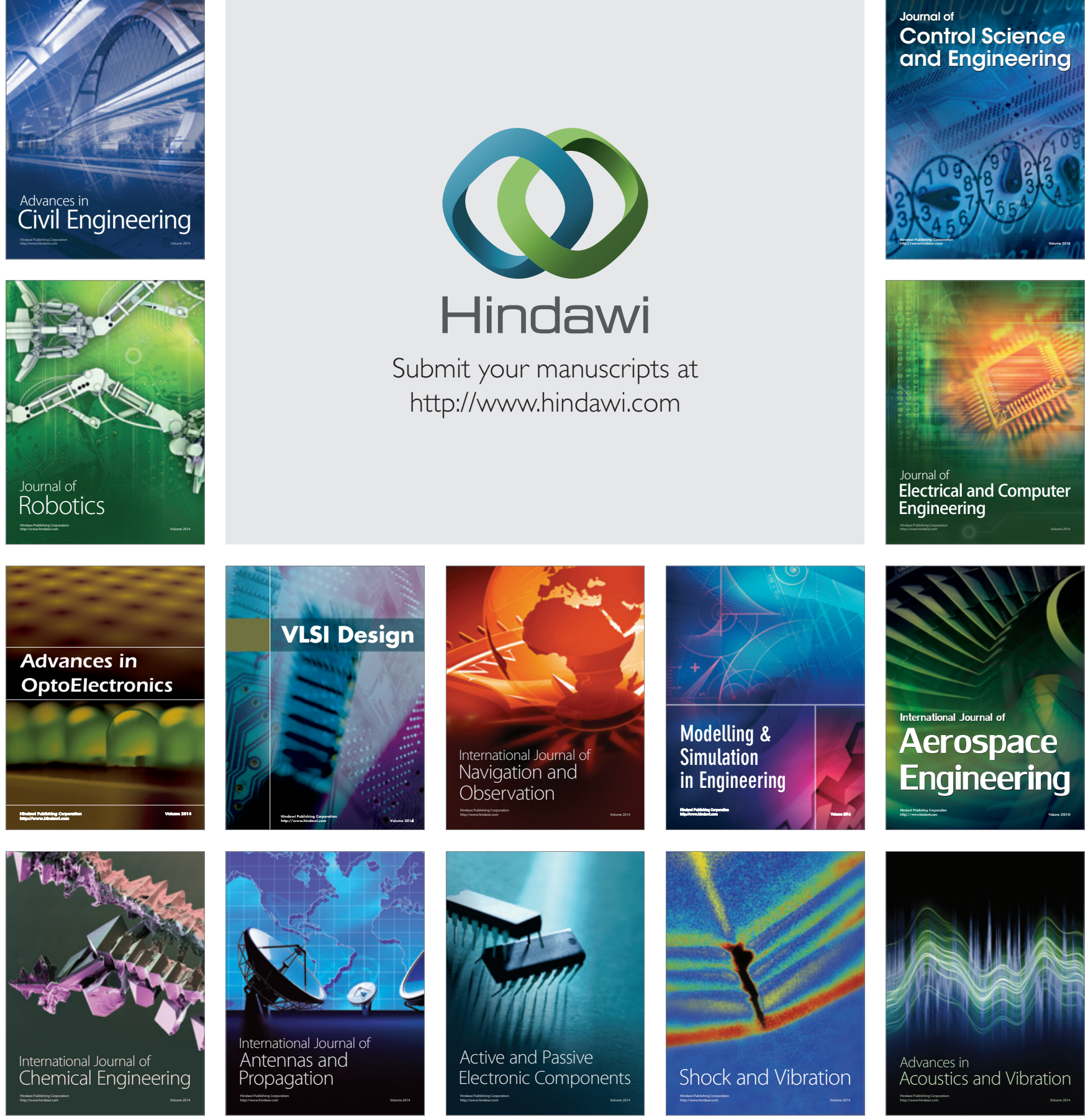